\title{
QUANTUM CONFINEMENT IN CdSe NANOCRYSTALLITES
}

\author{
K. E. Andersen, C. Y. Fong, W. E. Pickett \\ Department of Physics, University of California, Davis, CA 95616-8677, USA
}

\begin{abstract}
Quantum confinement increases the spacing between energy levels as the nanocrystallite size is decreased. Its qualitative features hold both for states localized near the center of a nanocrystallite and those near the surface, such as states due primarily to dangling bonds. However, different quantitative features are expected because of the different size constraints on each of these states. Since the majority of atoms in a typical nanocrystallite are on the surface, contrasting confinement effects between these two types may prove useful in predicting how surface state dependent properties, such as optical absorption, change with the size of the nanocrystallite. By applying first principles pseudopotential methods to indium doped, uncapped CdSe nanocrystallites containing 17 and 34 atoms, we identify center and surface localized states. Using the lowest occupied energy state as a reference, the energy of a state localized near the center is found to increase $24 \mathrm{mRy}$ from the 34 to 17 atom nanocrystallite. An equivalent surface state within the two cases studied is not found, but the energy level spacing is speculated to increase on the order of 100 mRy between the 34 and 17 atom cases based on states that are highly local to the surface, but not equivalent. Furthermore, we find it's necessary for the impurity to sit at the center of the nanocrystallite in order for the impurity states to be electrically active.
\end{abstract}

Preprint submitted to Elsevier Science

5 November 2018 
Key words: Colloids, Doping, Impurities, Localization

PACS: C220, D230, I130, L200

\section{Introduction}

The origin of quantum confinement in so-called zero-dimensional nanocrystallites, such as quantum dots (QDs), is understood to arise from the spatial confinement of electrons within the crystallite boundary. It leads to a larger spacing between energy levels as the size of the nanocrystallite is decreased. Qualitatively this effect is analogous to the problem of a particle in a box, and efforts to quantify confinement effects have been the topic of considerable research[1]. In CdSe semiconductor QDs, an important consequence of quantum confinement is the increase in the band gap as the QD size is decreased. Since this is observed as an increase in the energy of the lowest exciton peak as the radius of the $\mathrm{QD}$ is decreased[2,3], research in this area has focused almost exclusively on understanding the energy spectrum of an exciton as a function of $\mathrm{QD}$ radius $[1,4,5]$ in order to predict the optical properties of $C d S e$ QDs of an arbitrary size. However, in principle, quantum confinement should affect every electronic state within the QD, but not equally. For instance, electrons within a nanocrystallite can be confined in different spatial regions, such as near the center or surface, and these different regions should lead to discernible differences in how the spacing between energy levels changes with respect to the size of the QD.

Using first principles self-consistent pseudopotential methods, we quantify these differences for the electronic states within small, uncapped CdSe QDs containing 17 and 34 atoms ( $1 \mathrm{~nm}$ diameter). Doped $C d S e$ quantum dots are 
examined, with indium (a donor) substituted for cadmium, allowing distinct impurity states to be identified. We contrast confinement effects for impurity states confined near the center of the QD to those confined near the surface. Furthermore, we find that placing the impurity at the center of the $\mathrm{QD}$ is necessary for these impurity states to be electrically active.

Contrasting confinement effects between states localized near the center to those localized near the surface is important, since the majority of atoms within a QD are located on the surface. Quantifying quantum confinement in nanocrystallites, such as QDs, requires refinement of the particle in a box picture to incorporate the different "boxes" available within a nanocrystallite; in particular the difference between the surface and center.

\section{Models}

Two sizes of $C d S e$ QDs containing 17 and 34 atoms were modeled (Fig. 1). These were constructed using the cubic zincblende crystal structure up to a cutoff radius of 8.35 and 11.81 a.u., for the 17 and 34 atom cases respectively, from a central $C d$ atom. This structure was then surrounded within a supercell of 30.0 and 40.0 a.u. respectively, on each side. No relaxation of the ionic positions was done, since such relaxation can itself be a formidable task and the effect we are studying should not depend strongly on it.

These models represent uncapped, non-interacting collodial $C d S e$ quantum dots with diameters of roughly $1 \mathrm{~nm}$. To make the confining region at the surface similar between the two cases, an atom was removed from the 34 atom QD to make the bonding environment at the selected surface site more like 
that of the 17 atom case (shown in Fig. 1). Without this atom, both the 17 and 34 atom cases have a site on the surface that is missing three bonds. Indium was then substituted for cadmium at either this site or the center.

\section{Methods}

\subsection{Electronic Properties}

Norm-conserving pseudopotentials of the Hamann form[6] were used in con-

junction with the local density approximation of density functional theory $[7,8]$ to calculate all electronic properties. The wave function was expanded in a plane wave basis with a 50.0 Ry energy cutoff, using approximately 150,000 and 375,000 plane waves for the 17 and 34 atom cases respectively.

\subsection{Identifying States}

The added electron associated with the indium atom introduces a unique impurity state that, when substituted at either the center or a surface site, provides a way to identity a particular state between QDs of different size that will be localized either near the center or surface. By calculating selfconsistently the wave function and energy spectrum using the local density approximation of density functional theory it is possible to 1) identify these impurity states by analyzing the projected density of states local to an atom and 2) quantify the confinement effect on these states, using the lowest energy state as a reference. 
The projected density of the $n$th state $D_{n}(\epsilon, r, \vec{\tau})$ local to an atom at the location $\vec{\tau}$ is used to identify states.

$$
\begin{aligned}
D_{n}(\epsilon, r, \vec{\tau}) & =\frac{1}{V} \sum_{l=0}^{\infty} \sum_{m=-l}^{l}\left|\left\langle l m \mid \psi_{n}(\vec{r}-\vec{\tau})\right\rangle_{a n g}\right|^{2} \delta\left(\epsilon-\epsilon_{n}\right) \\
& =\frac{1}{V} \sum_{l=0}^{\infty} \sum_{m=-l}^{l}\left|4 \pi \sum_{\vec{G}} a_{\vec{G}, n} \mathrm{e}^{-i \vec{G} \cdot \vec{\tau}} j_{l}(G r) Y_{l m}^{*}\left(\theta_{\vec{G}}, \phi_{\vec{G}}\right)\right|^{2} \delta\left(\epsilon-\epsilon_{n}\right)
\end{aligned}
$$

Here $\psi_{n}$ and $\epsilon_{n}$ are the wave function and energy of the $n$th state, and $V$ is

the volume of the unit cell. $\psi_{n}(\vec{r}-\vec{\tau})=\sum_{\vec{G}} a_{\vec{G}, n} \mathrm{e}^{i \vec{G} \cdot(\vec{r}-\vec{\tau})}$ is projected onto the spherical harmonic basis $|l m\rangle$. Based on the magnitude of the $s(l=0), p$ $(l=1)$, and $d(l=2)$ components it is possible to identify the states associated with an atom, in particular, the impurity.

\section{Results}

The lowest energy state is used as a reference to compare energies between QDs of different size and impurity location. This state, which can be thought of as originating from the $4 \mathrm{~s}$ state of the interior shell of $\mathrm{Se}$ atoms, was selected because it is expected to be the most inert and therefore least affected by the addition of the impurity atom. Fig. 2 shows the projected density, as defined in Eq. 1, of the lowest energy state for the cases considered. The projection origin $\vec{\tau}$ is the center of the QD, which corresponds to either a $C d$ or In atom. With the projection oriented as such, the $s$ character shown is a result of a symmetric combination of orbitals on each of the four neighboring, tetrahedrally positioned Se atoms. No substantial $p$ or $d$ components are present for this state. For the case of In at the center of the QD (the middle column of graphs), the more attractive In pseudopotential (compared to $C d$ ) 
distorts these orbitals leading to an increase in the peak between the $I n$ and Se atoms at approximately 2 a.u..

The assumption of the lowest energy state being inert holds for the case of the impurity at the surface of the quantum dot, which only differs in energy from the lowest energy state with no impurity by 0.7 and $5.7 \mathrm{mRy}$ for the 17 and 34 atom cases. To within the accuracy of our calculations, which are on the order of $1 \mathrm{mRy}$, these two states are essentially identical in energy.

However, the assumption doesn't hold when the impurity is at the center. The energy difference between the lowest energy state with the impurity at the center and the lowest energy state with no impurity is $31.7 \mathrm{mRy}$ for the 17 atom case and $20.2 \mathrm{mRy}$ for the 34 atom case. We attribute this non-constant shift in energy of the lowest energy state between QDs of different size to quantum confinement on the lowest state. For the impurity at the surface, the effect of quantum confinement is negligible; however, when the impurity is at the center of the QD there is a $11.5 \mathrm{mRy}$ discrepancy in the shift of the lowest energy state.

With the impurity at either the center or a surface site, the projected density is analyzed to identify an analogous state between the 17 and 34 atom quantum dots. The 34 atom case, because it has more electrons, necessarily has more electronic states. However, if a state is found within its energy spectrum that is similar in character to a state in the energy spectrum of the 17 atom QD, then these states can be considered to be physically the same. Comparing the energies of such a state with respect to a suitable reference will then quantify the effect due to confinement. 


\section{Discussion}

For the case of the impurity at the center (Fig. 3), it was possible to identify a unique state between the two sizes of QDs. For the 17 atom case, this state was the lowest unoccupied energy state and is $1.0038 R y$ above the lowest energy state of the 17 atom QD. For the 34 atom case, it was the highest occupied energy state at an energy of $0.9685 R y$ above the lowest state. Naïvely, this suggests that quantum confinement has increased the energy level spacing for this state by $35 \mathrm{mRy}$ as the size of the QD was reduced from 34 to 17 atoms. However, because the lowest energy state of the 17 atom case has itself been affected by confinement this value should instead be $24 \mathrm{mRy}$.

With the impurity at the surface, finding a similar state between the two sizes of QDs is complicated by the surface; even with the removal of one atom from the surface to make the impurity sites similar, as discussed in $\S 2$ and shown in Fig. 1. In contrast to the case of the impurity at the center, no states near the highest occupied energy state were significantly localized near the impurity atom. However, there were significantly localized states below this energy level. An example of two such states is shown in Fig. 4. Physically, the impurity state at the surface is more bound than the corresponding impurity state at the center. At the center the bonding with the nearest neighbor layer of $S e$ is complete with $C d$. Substituting In for $C d$ introduces an extra electron that is relatively unlocalized. This is supported by the predominantly $s$ projected density seen in Fig. 3. At the surface, however, the nearest neighbor layer of $S e$ has only three neighboring $C d$ atoms, instead of four. The extra electron introduced when In is substituted for $C d$ therefore participates in bonding, resulting in a lower energy for that state. 
Since the identification of a unique impurity related surface state for both sizes of QD isn't possible, we proceed by comparing two states which are localized near the impurity atom, but not equivalent. The projected density of the chosen states is in Fig. 4. Other states were also found to be localized around the impurity. The selected states were chosen because they were the most localized states near the highest occupied energy state. For the 17 atom case the chosen state is at an energy of $0.778 R y$ above the lowest occupied energy state. Within the energy spectrum of the 34 atom case, the chosen state is at an energy of $0.6530 R y$ above the lowest occupied energy state of that system. To compare, the spacing between the energy level of the chosen state and the lowest occupied energy state has increased on the order of 100 mRy between the 34 and 17 atom QDs.

The observation that confinement effects at the surface are more pronounced than in the center of the QD is suggestive, but hardly convincing based on the ad hoc assumption made that the localized states chosen could be compared between the two sizes of QDs. In ongoing research we are investigating the charge density of these states to see if a more certain identification can be made. In addition, we're looking at a 71 atom QD model (essentially another layer of $S e$ and $C d$ ) that mimics the surface bonding environment of the 17 atom case much more closely, with the hope of eliminating this ambiguity.

\section{Conclusion}

In identifying a reference energy, which is a necessary prerequisite of any quantitative analysis of quantum confinement, we encountered the complication that the most suitable energy level, the lowest occupied energy state, was 
itself affected by quantum confinement. By making a comparison to the case with no impurity, it was possible to quantify this discrepancy to find that the energy level spacing for states localized near the center of the QD increased 24 mRy between the 34 and 17 atom cases. For states localized near the surface, although identifying a suitable reference state was straightforward, unambiguously identifying a state near the surface in both the 17 and 34 atom cases was impossible due to complications at the surface. Our results suggest that the energy level spacing of such a state would increase on the order of 100 $m R y$ in going from the 34 to the 17 atom QD. Furthermore, in identifying impurity states we found that doping at the center was necessary to introduce electrically active impurity states. Such states, either at or just above the highest occupied energy level, would be expected to contribute to transport and optical properties within these systems.

\section{Acknowledgements}

KEA acknowledges support from the UC Davis IGERT Program on Nanophases,

NSF Grant No. DGE-9972741. CYF acknowledges support from NSF Grant No. INT-9872053.

\section{References}

[1] A. D. Yoffe, Low-dimensional systems: quantum size effects and electronic properties of semiconductor microcrystallites (zero-dimensional systems) and some quasi-two-dimensional systems, Advances in Physics 42 (2) (1993) 173266 , and references therein. 
[2] D. J. Norris, A. L. Efros, M. Rosen, M. G. Bawendi, Size dependence of exciton fine structure in CdSe quantum dots, Physical Review B 53 (24) (1996) 16347-54.

[3] P. A. M. Rodrigues, G. Tamulaitis, P. Y. Yu, S. H. Risbud, Size selective photoluminescence exitation spectroscopy in CdSe nanocrystals, Solid State Communications 94 (8) (1995) 583-7.

[4] L.-W. Wang, A. Zunger, Pseudopotential calculations of nanoscale CdSe quantum dots, Physical Review B 53 (15) (1996) 9579-82.

[5] K. Leung, K. B. Whaley, Surface relaxation in CdSe nanocrystals, Journal of Chemical Physics 110 (22) (1999) 11012-22.

[6] D. R. Hamann, Generalized norm-conserving pseudopotentials, Physical Review B 40 (5) (1989) 2980-87.

[7] P. Hohenberg, W. Kohn, Inhomogeneous electron gas, Physical Review 136B (3) (1964) 864-71.

[8] W. Kohn, L. J. Sham, Self-consistent equations including exchange and correlation effects, Physical Review 140A (4) (1965) 1133-38. 


\section{List of Figures}

1 Models of CdSe quantum dots containing 17 (top) and 34

(bottom) atoms. (Not to scale.) Both quantum dots have a

diameter of approximately $1 \mathrm{~nm}$ and are centered around a

cadmium atom. The dashed line illustrates schematically the

30.0 and 40.0 a.u. supercell, for the 17 atom and 34 atom cases

respectively. Indium is substituted for cadmium at either the

center or the surface site shown.

$2 \quad$ Projected density for the lowest energy state, with the projection origin at the center of the QD. The columns, from

left to right, are for the case with no impurity, the impurity at the center, and the impurity at the surface for the 17 atom QD (graphs along the top row) and 34 atom QD (bottom row). The location of the neighboring $S e$ atom is shown by a line at 5.11 a.u.. The energy of the state is labeled.

3 The projected density for two equivalent states in the 17 (top) and 34 (bottom) CdSe quantum dot models. These states, at the lowest unoccupied (17 atom case) and highest occupied (34 atom case) energy states, are localized spatially near the center of the quantum dot. The relative energy of the state is given with respect to the lowest occupied energy level of the system. 
$4 \quad$ Projected density for states that are localized near the impurity atom $(\operatorname{In})$ at the surface of the 17 (top) and 34 (bottom) atom QDs. Both of these states are lower in energy than the highest occupied energy level. The relative energy of the state is given with respect to the lowest occupied energy level of the system. 


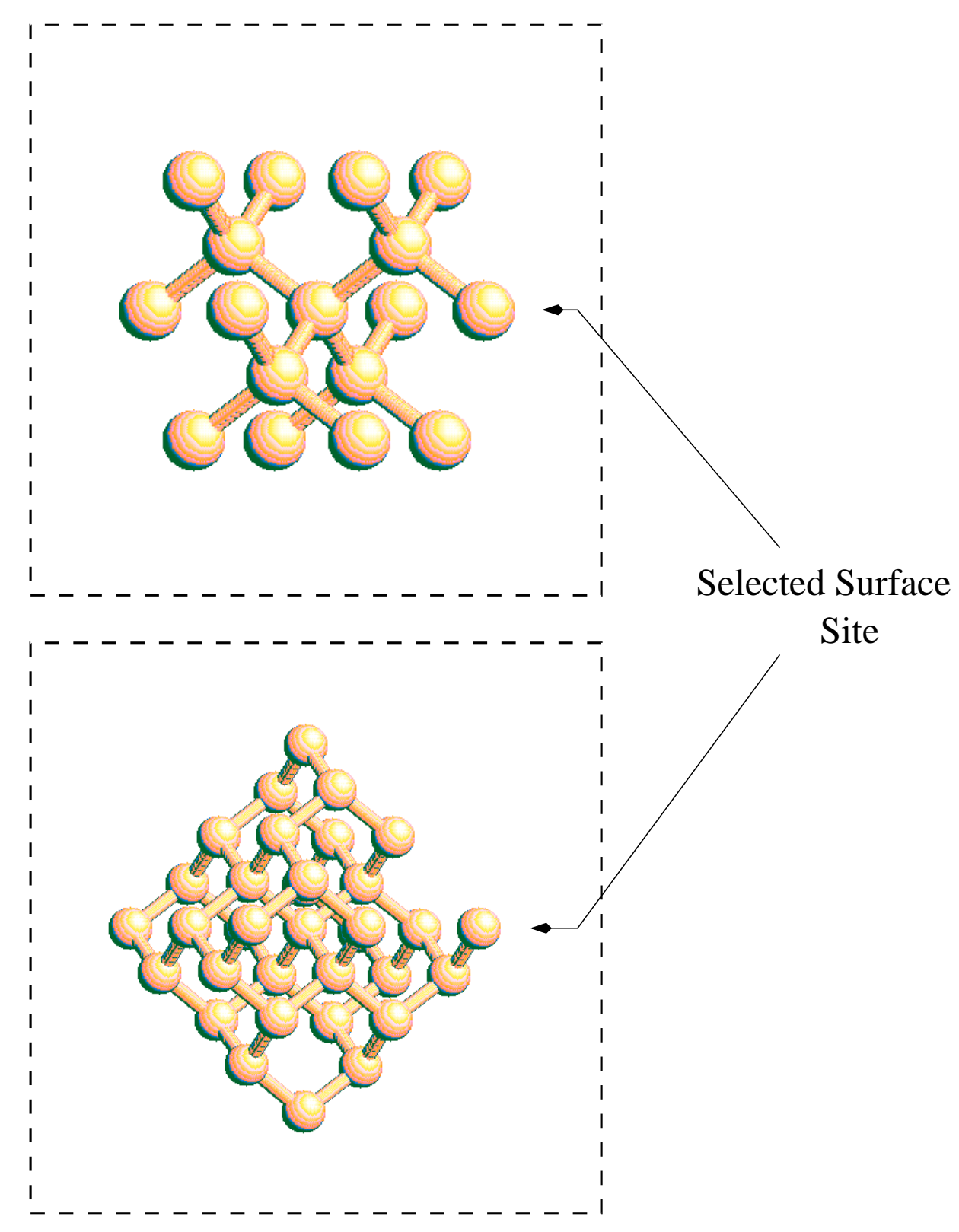

Fig. 1. 


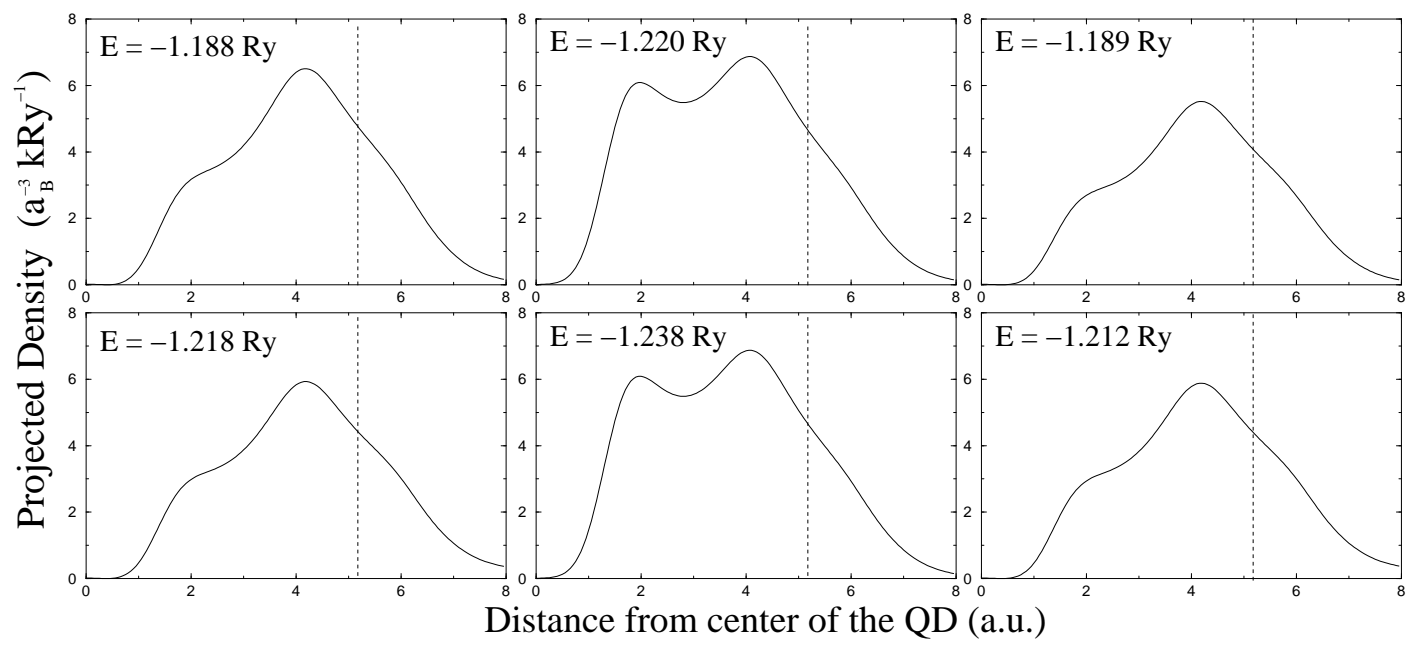

Fig. 2.

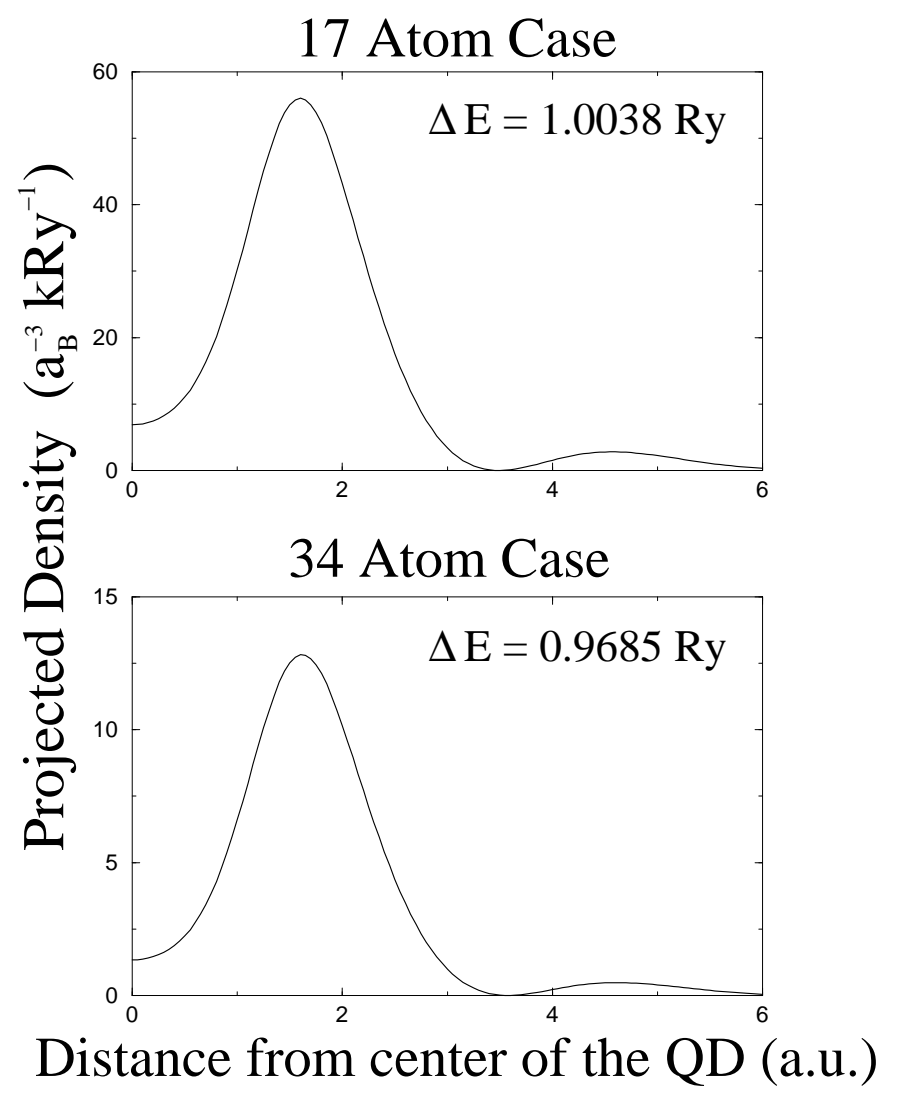

Fig. 3. 


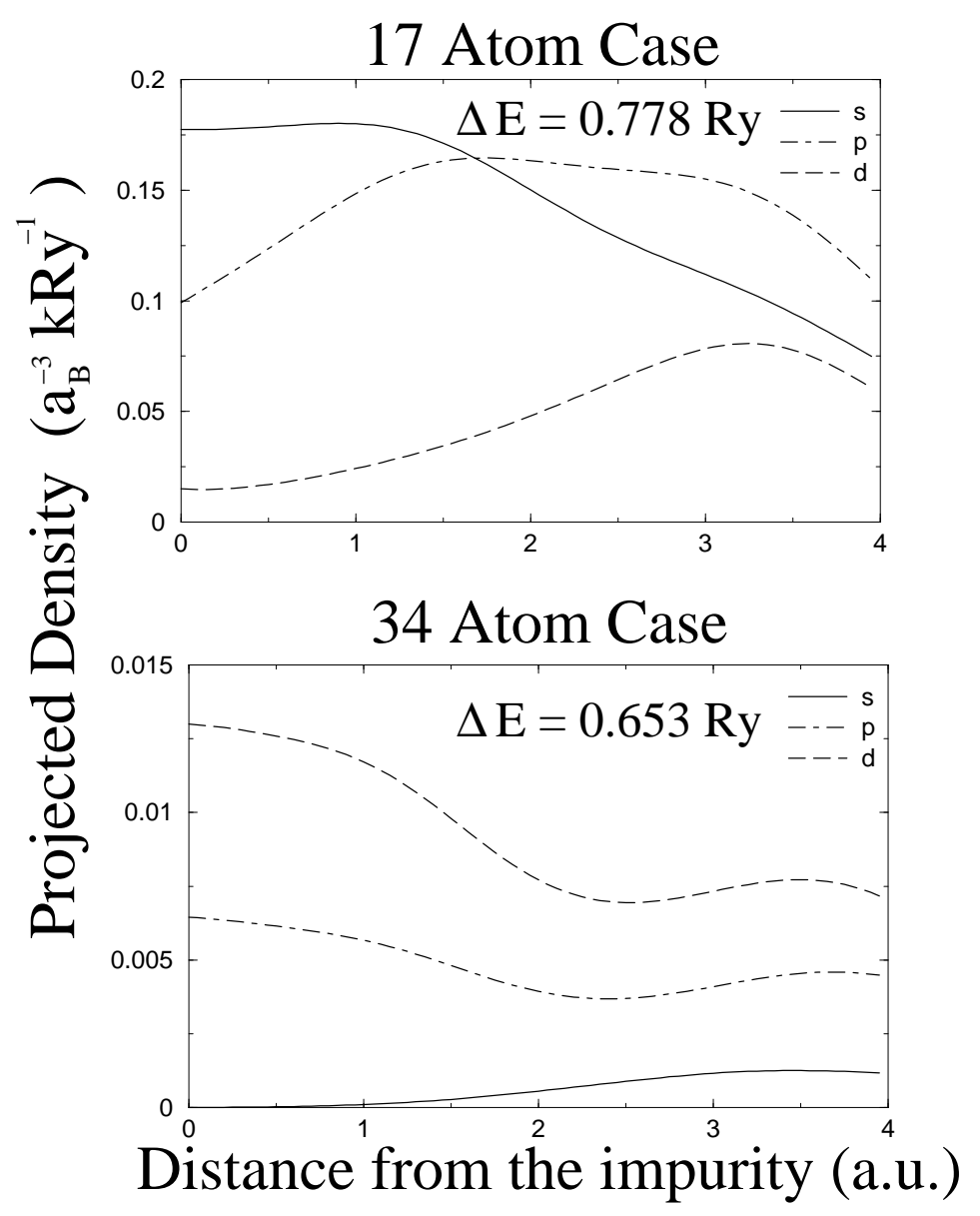

Fig. 4. 